LBL-36895

UC-414

Presented at the Strangeness '95 Workshop,

Tucson, Arizona, January 4-6, 1995, and

Proceedings will be published by the American Institute of Physics.

\title{
Strangeness Production as a Function of Centrality
}

\author{
Douglas E. Greiner \\ Lawrence Berkeley Laboratory \\ University of California, Berkeley, CA 94720
}

and

The NA36 Collaboration

February 1995

This work was supported by European Community Grant A8000145; the Director, Office of Energy Research, Division of Nuclear Physics of the Office of High Energy and Nuclear Physics of the U.S. Department of Energy under Contract DE-AC03-76SF00098 and DE-FG02-91ER40652; United Kingdom Science and Engineering Research Council Grant GR/H 71314; Norway Grant Norges Forskningsråd (NAVF); Spain under CICYT Contracts 85-0022, AE86-0031, AE87-0031, AE88-0031, AEN90-0739 and XUG 80409288.

$-x$ 


\section{DISCLAIMER}

This report was prepared as an account of work sponsored by an agency of the United States Government. Neither the United States Government nor any agency thereof, nor any of their employees, make any warranty, express or implied, or assumes any legal liability or responsibility for the accuracy, completeness, or usefulness of any information, apparatus, product, or process disclosed, or represents that its use would not infringe privately owned rights. Reference herein to any specific commercial product, process, or service by trade name, trademark, manufacturer, or otherwise does not necessarily constitute or imply its endorsement, recommendation, or favoring by the United States Government or any agency thereof. The views and opinions of authors expressed herein do not necessarily state or reflect those of the United States Government or any agency thereof. 


\section{DISCLAIMER}

Portions of this document may be illegible in electronic image products. Images are produced from the best available original document. 


\title{
Strangeness Production as a Function of Centrality
}

\author{
Douglas E. Greiner for the NA36 Collaboration
}

E. Andersen ${ }^{1}$, R. Blaes ${ }^{2}$, M. Cherney ${ }^{3}$, B. de la Cruz C. Fernández $^{5}$, C. Garabatos ${ }^{5}$, J.A. Garzón ${ }^{5}$, W.M. Geist ${ }^{2}$, D.E. Greiner ${ }^{6}$, C.

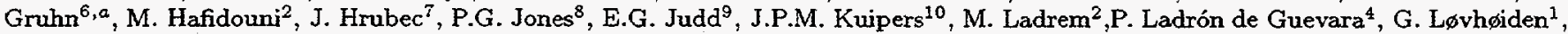
J. MacNaughton ${ }^{7}$, J. Mosquera ${ }^{5}$, Z. Natkaniec ${ }^{10}$, J.M. Nelson ${ }^{8}$, G. Neuhofer ${ }^{7}$, C. Pérez de los Heros ${ }^{4}$, M. Pló ${ }^{5}$, P. Porth ${ }^{7}$, B. Powell ${ }^{10}$, A, Ramil ${ }^{5}$, H. Rohringer ${ }^{7}$, I. Sakrejda ${ }^{6}$, T.F. Thorsteinsen ${ }^{1}$, J. Traxler $^{7}$, C. Voltolini ${ }^{2}$, K. Wozniak ${ }^{11}$, A. Yañez ${ }^{5}$, and R. Zybert ${ }^{8}$ NA36 mailing address: NA36, c/o Dr. D. E. Greiner, Mailstop 50D, Lawrence Berkeley Laboratory, 1 Cyclotron Road, Berkeley CA 94720, USA.

\author{
${ }^{1}$ University of Bergen, Dept. of Physics, N-5007 Bergen, Norway \\ ${ }^{2}$ Centre de Recherches Nucléaires, IN2P3.CNRS/Université L. Pasteur, BP 20, F-67037 Strasbourg, France \\ ${ }^{3}$ Creighton University, Dept. of Physics, Omaha, Nebraska 68178, USA \\ ${ }^{4}$ CIEMAT, Div. de Física de Particulas, E-28040 Madrid,Spain \\ ${ }^{5}$ Universidad de Santiago, Dpto. Física de Particulas, E-15706 Santiago de Compostela, Spain \\ ${ }^{6}$ Lawrence Berkeley Laboratory (LBL), University of California, Berkeley CA 94720, USA \\ ${ }^{7}$ Institut für Hochenergiephysik (HEPHY), A-1050 Wien, Austria \\ ${ }^{8}$ University of Birmingham, School of Physics and Space Research, Birmingham B15 2TT, UK \\ ${ }^{9}$ University of California Space Sciences Laboratory, Berkeley CA 94720, USA \\ ${ }^{10}$ European Organization for Nuclear Research (CERN), CH-1211 Genève 23, Switzerland \\ ${ }^{11}$ Instytut Fizyki Jadrowej, PL-30 055 Krakow 30, Poland \\ a Present address: PPE division, CERN, Geneva, Switzerland
}

\begin{abstract}
A correction to the TPC efficiency calculations based on discriminator response data is shown to have an average effect of $20 \%$ on the absolute magnitude of the earlier results. Consistency between runs with the NA36 magnet in different polarities is demonstrated. Comparisons are made with NA35 S + Ag data. The absolute flux of $\Lambda$ particles is approximately a factor of two in disagreement between the two experiments. A dependence of the rapidity spectrum for lambda's on centrality is demonstrated.
\end{abstract}

\section{EFFICIENCY CALCULATIONS}

In general large solid angle tracking experiments need to have an accurate monte carlo simulation in order to make the corrections necessary to produce device independent physics measurements. This is true for all imperfect tracking systems such as TPC's, streamer chambers and MWPC's. The high multiplicity environment of relativistic heavy ion collisions makes the monte carlo more important while at the same time making the task more difficult. Tracks will merge and cross, the efficiency to find them becomes not only a function of the resolution of the hardware but also a function of the tracking software used to search for them. The NA36 monte carlo uses GEANT to simulate the particles moving through the detector and special routines for simulating the response of the TPC to their passage. The TPC response routines are tuned to reproduce the measured basic properties of the detector such as hit efficiency and spatial resolution. Secondary quantities such as momentum resolution, chi-square distributions, etc. etc. were also checked to ascertain that the monte carlo data had the same properties as the real data.

NA36 used a standard method to calculate the efficiency of finding the $\mathrm{V}^{0}$ topology. This was to generate a $\mathrm{V}^{0}$ that can be found in the TPC and embed it in actual data. When this is done one needs to know in detail how the detector responds to tracks which may be close to each other. In the NA36 TPC (see Fig. 1) the spacing of the short anode wires determines the track pair resolution in the bend plane, this is straight forward to simulate. However, in the drift direction the track merging is a function of the response of the electronics to a pair of pulses arriving at the anodes close or overlapping in time. In mid 1994 we decided to make some measurements to verify our assumptions about the response of our electronics. The TPC [1] was used in the configuration we used for data taking in 1990 , stimulated by a $\mathrm{Sr}^{90}$ source and pulse shapes were recorded for pulses entering the discriminator and the corresponding discriminator output logic signal. The expected behavior of the discriminator is shown in Fig. 2 . When the threshold intersects the pulse on both the leading and trailing edge the discriminator is triggered to begin and end the logic pulse indicating signal over threshold is present. The reason the pulse shape measurements were done was to examine the shape of the pulses when they were below threshold. Their shape then determines what the response is when tracks just begin to overlap. Our model at this point did not allow pulses to overlap till the actual discriminator pulses overlapped. We wanted to see if there was an appreciable effect on the efficiency if these pulse tails were properly included in the calculation. The actual behavior of the discriminator for small pulses was 


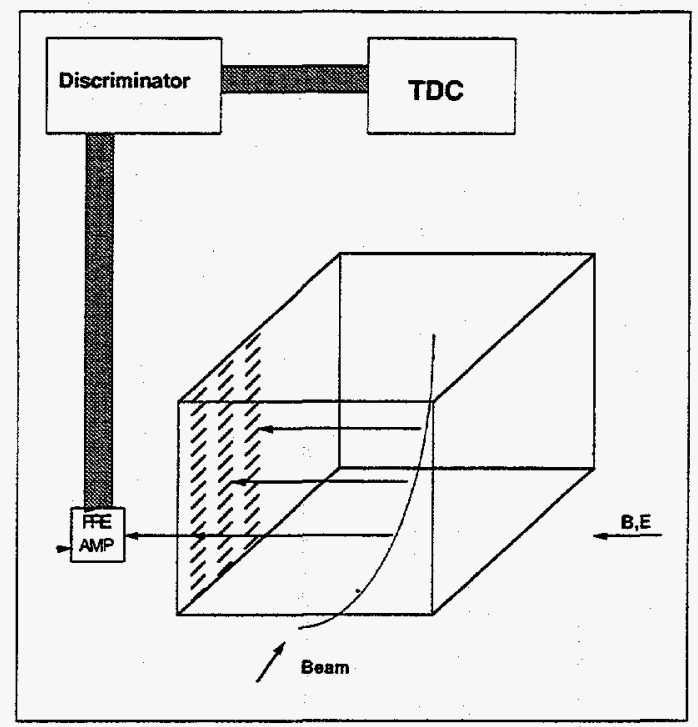

FIG. 1. NA36 TPC and electronics. Beam goes below the TPC. The signal in the bend plane is detected by rows of anode wires on the endcap. Position in the drift direction is determined by the drift time to the endcap. The preamps were LeCroy TRA402 and the discriminators were LeCroy MVL407. Pulse shaping was done before the discriminator. The TDC's digitized the time and length of the discriminator pulses in $25 \mathrm{~ns}$ bins.

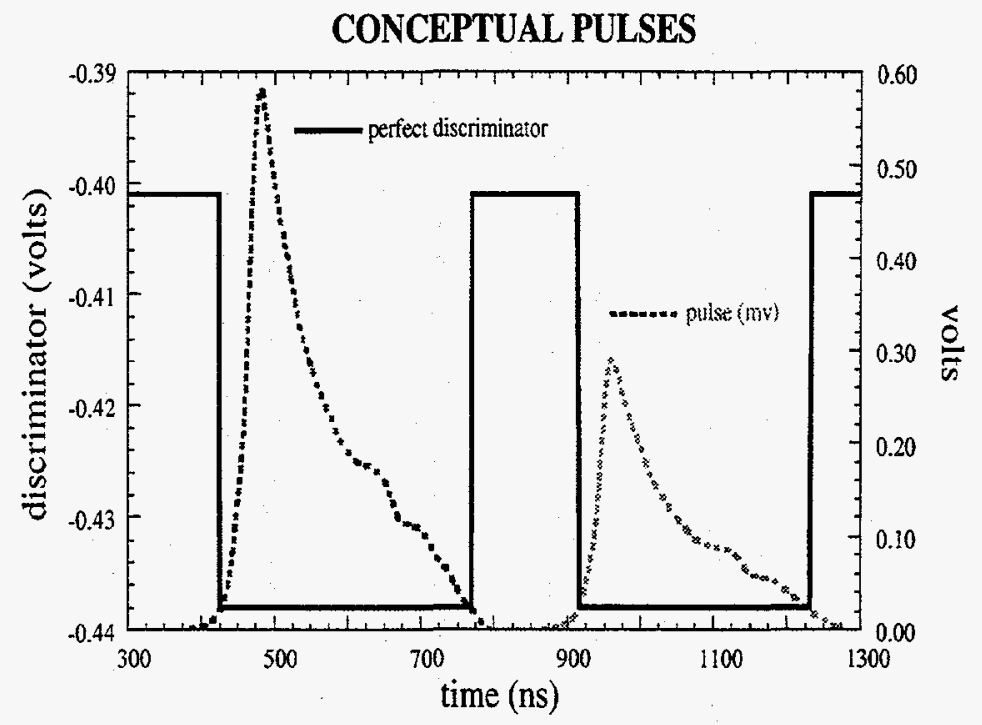

FIG. 2. Perfect discriminator responding to two pulses. Pulses are actual shapes produced by the electronics. 


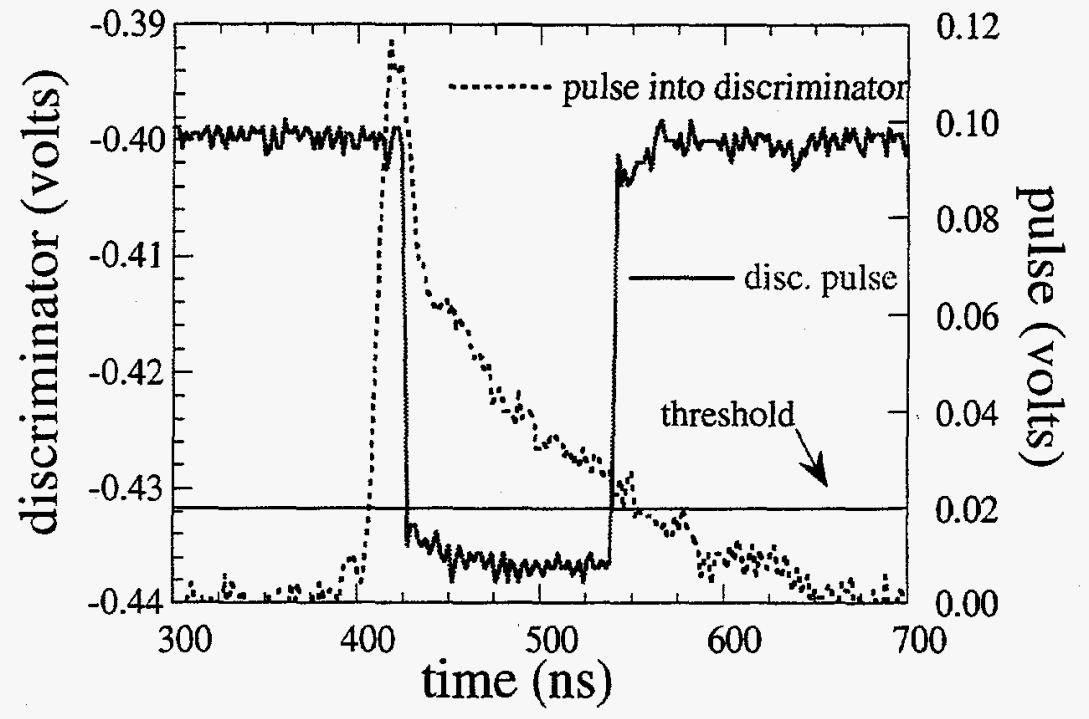

FIG. 3. Discriminator response to a small pulse. There is a slight delay of about 25 ns before discriminator responds to the leading edge of the pulse.

close to the assumed response as shown in Fig. 3. However there is a delay of about $25 \mathrm{~ns}$ before the discriminator responds to the leading edge of the pulse. For larger pulses, see Fig. 4, our tests revealed an effect we had not anticipated. The trailing edge response of the discriminator was coming at a higher value of the pulse than indicated by the known threshold setting, producing a shortening of the signal length. In order to quantify the characteristics of the discriminator response we examined a range of pulse heights. As the TPC electronics produces only start point and time-over-threshold we first verify that the pulse height and time-over-threshold are well correlated as shown in Fig. 5a. In Fig. 5b we see that the leading edge trigger delay is constant with pulse length. The major problem to deal with is then seen in Fig. $5 \mathrm{c}$, the strong rise in the effective threshold for the trailing edge of the pulses. We need to determine how much this defect affects the efficiency calculations for finding $\mathrm{V}^{0}$ 's. First we look to the physics data to see the magnitude of the effect. If we assume that the threshold rises in response to the height of the recent pulses and then returns to the set value after some time range then pulses that are close together on the same anode wire would appear shorter than those alone or far apart. How frequently this happens in the data give guidance as to the size of the effect. In Fig. 6 is plotted the pulse length for pulses on the same anode wire and separated by $0-5 \mathrm{~cm}$, $6-10 \mathrm{~cm}$ and alone. From this figure we conclude that the pulses do indeed get shorter as they come closer together and that this happens about $1 \%$ of the time in the physics data. However a $1 \%$ effect is not to be neglected as the very $\mathrm{V}^{0}$ topology we are looking for involves tracks that emerge from a common vertex. They are therefore very close together till the bending in the magnetic field seperates them.(Fig. 11)

In order to model the pulse overlap we need to verify that the pulses can be scaled and added linearly. Fig. 7 shows a comparison of two pulses that are about a factor of 2.5 different in magnitude that are normalized to the same height. Comparing shapes we see that to within about $10 \mathrm{~ns}$ they agree. This is within the digitization size of our TDC's which is $25 \mathrm{~ns}$ so scaling the pulses is acceptable. To test the linear addition of pulses we make use of the data where we have several overlapping pulses in a single trigger, these events are invaluable to test models of the overlap process. In Fig. 8 we see a set of three overlapping pulses compared with a linear superposition of three single pulse shapes. The agreement is within the variability of the single pulses. This agreement implies that linear superposition of pulses at the discriminator input is a valid assumption.

The discriminator was assumed to have some hysterisis such that it would raise the threshold as charge arrived and then lower it back to the baseline. A typical behavior of this kind is expressed by the difference of two decaying exponentials. The threshold at time $t_{i}$ is:

$$
\text { threshold }\left(t_{i}\right)=\text { threshold }(t=0)+\sum_{j=0}^{j=i} p_{j}\left(e^{-\frac{t_{j}-t_{i}}{T_{1}}}-e^{-\frac{t_{j}-t_{i}}{T_{2}}}\right)
$$

where $\mathbf{P}_{j}$ is the pulse height at time $t_{j}$. The adjustable parameters are the decay times $T_{1}, T_{2}$ and the delay of firing on the leading edge. The best fit values of these parameters were determined by a least squares fit to the 


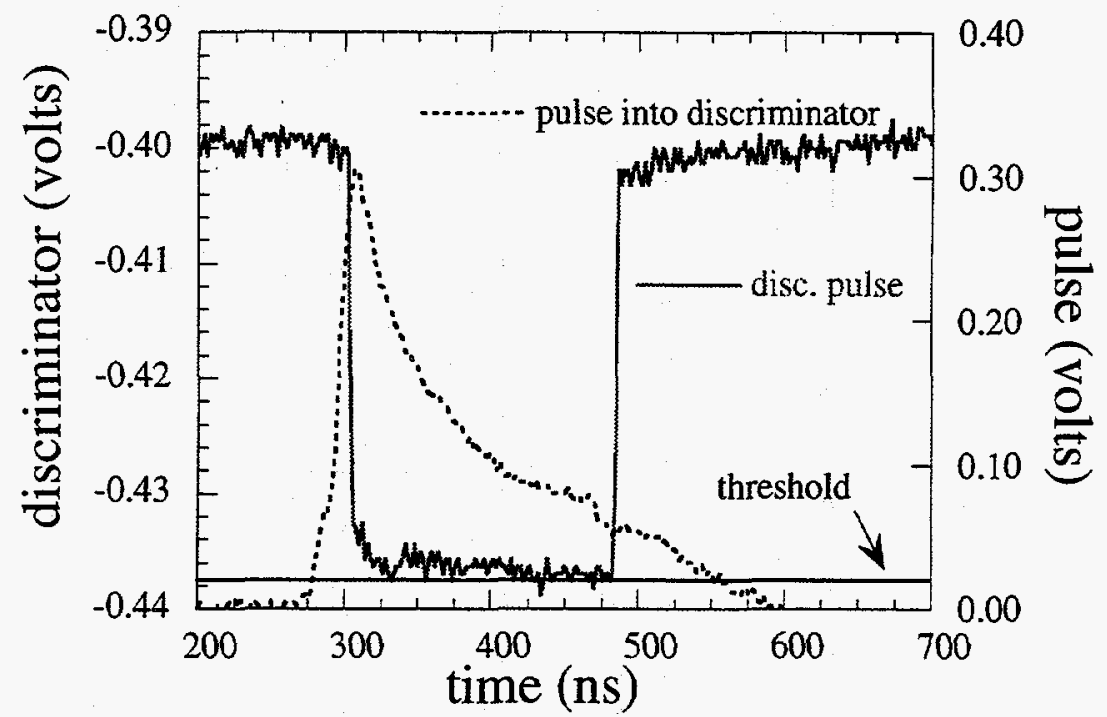

FIG. 4. Discriminator response to an average height pulse. Here we see that the discriminator response to the trailing edge of the pulse has triggered early.
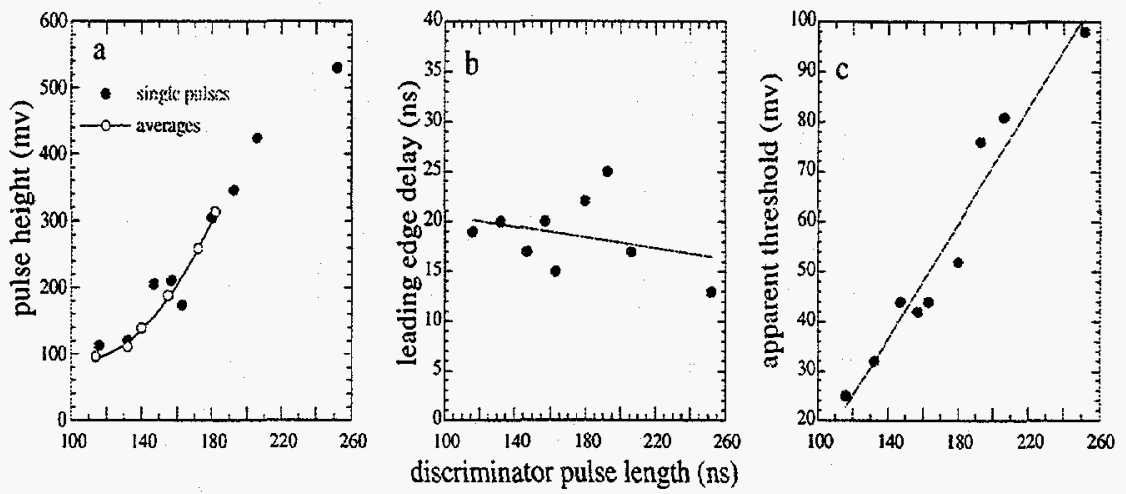

FIG. 5. Discriminator response to a range of pulse heights. a. Pulse height is well correlated with pulse length, it is pulse length that is measured in the TPC electronics, so this correlation is needed to parameterize the discriminator behavior. $b$. The leading edge delay is almost independent of the pulse length. c. The apparent threshold on the trailing edge of the pulses grows linearly with the pulse length. 


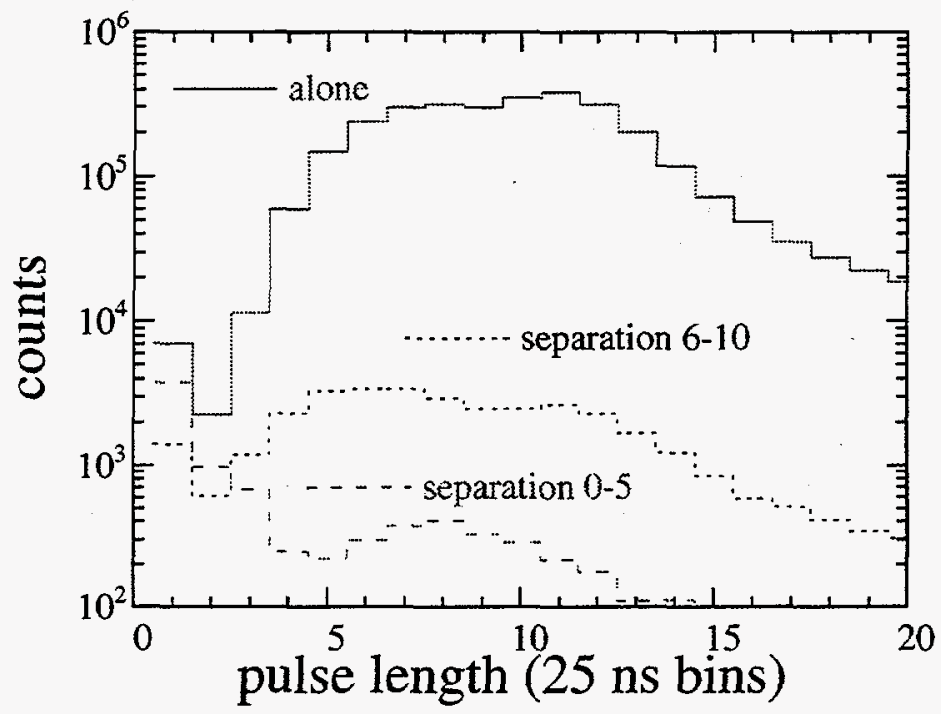

FIG. 6. Dependence of pulse length on pulse separation in physics data. About $1 \%$ of all pulses are close enough together on the same wire to have some shortening.

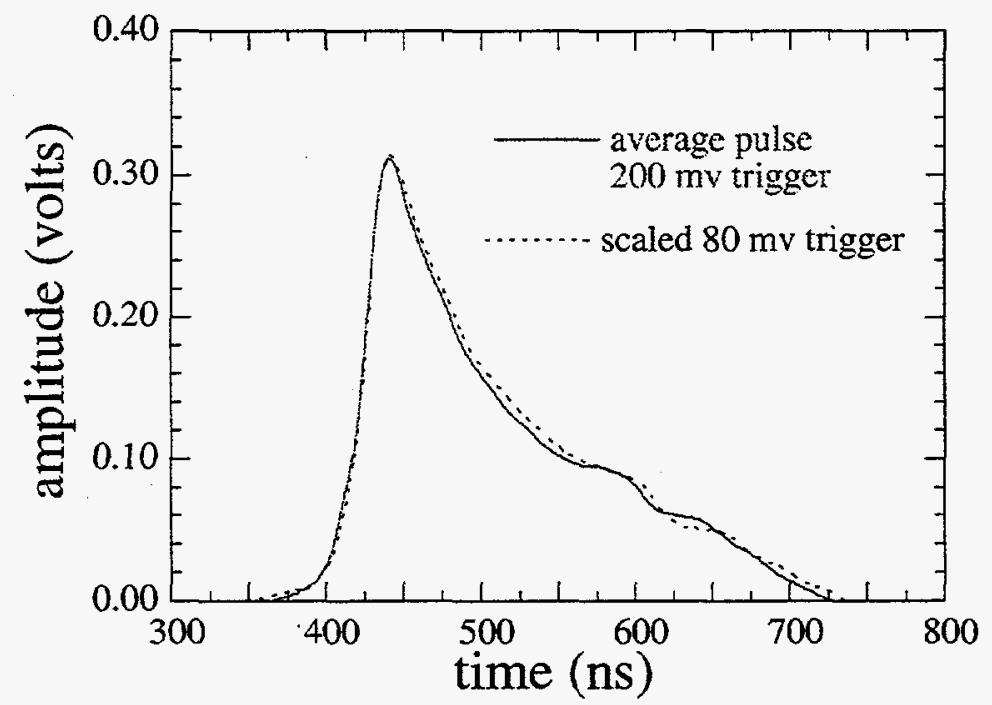

FIG. 7. Test of pulse shape dependence on amplitude. The digitization bin size of the physics data is the size of the tic marks on the time axis (25 ns). The pulses scale reasonably for our accuracy. 


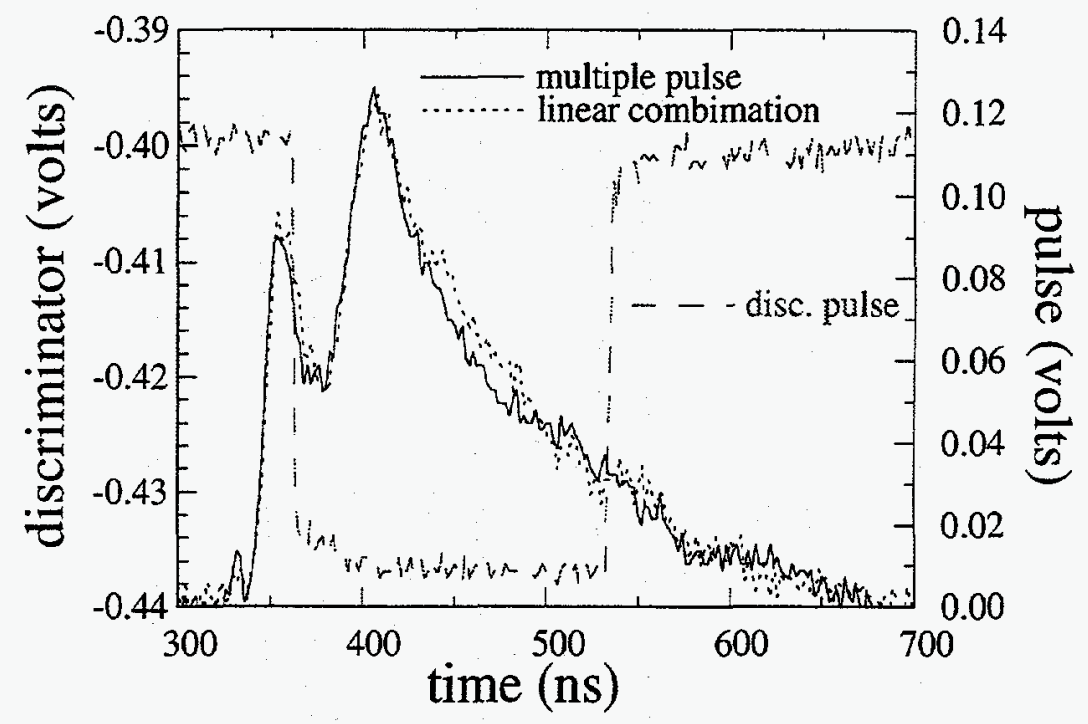

FIG. 8. Comparison of observed multiple pulses with a linear combination of one pulse shape adjusted to have same peak position and amplitude. The linear superposition is an acceptable fit to the multiple pulse data. Also shown is the discriminator response to the multiple pulse input.

discriminator response of 28 single and multiple pulse data sets. The best fit values were: $d=28 \pm 2 n s, T_{1}=156 \pm 17 n s$, $t_{2}=113 \pm 17 n s$. RMS error was $8 \mathrm{~ns}$, well within our digitization bin size.

Demonstrated in Fig. 9 is the model discriminator pulse vs the measured response for multiple pulse data. This model gives a good description of the circuit response to overlapping tracks. The efficiency calculations were modified to use this discriminator response model. We used the average pulse shapes and scaled them to have the appropriate pulse lengths to match the digitization interval. When embedding pulses in data all pulses within the decay time of the discriminator movement were re-evaluated using the discriminator model. In Fig 10 we compare the old and new values for the multiplicity and rapidity dependence of the efficiency to detect $\Lambda$ particles in our data. This comparison is for an E0 trigger corresponding to $52 \%$ of the reaction cross section with the rapidity and $P_{t}$ intervals being $1.5<$ $\mathrm{y}<3.0$ and $0.6<P_{t}<1.6$, respectively.

The effect of the pulse overlap on the efficiency can be seen as an overall shift of about $6 \%$ due to the $V^{0}$ topology with an increase with $\Lambda$ rapidity as the opening angle becomes smaller and an increase with event multiplicity because of interference by adjacent tracks. Typical average change is seen to be about $20 \%$ decrease in efficiency.

\section{NA36 RESULTS}

As the efficiencies have changed we repeat a standard consistency test for NA36 data. The experiment is asymmetric because we detect only the particles in the area above the beam. When one reverses the field in the NA36 magnet the experiment becomes sensitive to the $\mathrm{V}^{0}$ topology in a completely different manner. This is demonstrated in Fig. 11 where we see that for negative polarity we have the pion from the $\Lambda$ decay leaving the chamber quickly while in the positive polarity case the efficiency is enhanced by having the pion travel up through the sensitive region of the TPC. The data for the two polarities were taken days apart and have different beam positions and chamber efficiencies, they represent essentially two independent measurements. Agreement between these two measurements is a strong test of the entire measurement and analysis chain of NA36. In Fig. 12 is shown the $\Lambda$ rapidity spectrum from the analysis of each polarity data. We consider this agreement as corroboration that our experiment is strongly internally consistent. Treating the two measurements as independent measures we plot in Fig. 13 the combined data and compare it with the result we presented at QM93. [2] The QM93 data were for a smaller transverse momentum window $0.6<P_{t}<$ 1.6 vs $0.4<P_{t}<1.8 \mathrm{GeV} / \mathrm{c}$ for the combined data. We have scaled the combined data using the known $P_{t}$ slope to the same $P_{t}$ range and also scaled by the ratio of the average multiplicity to account for a slight difference in trigger. The combined scaling factor was 0.55 . As expected from the change in efficiency shown earlier the current combined data is an average of $16 \%$ higher, the shape is the same. 


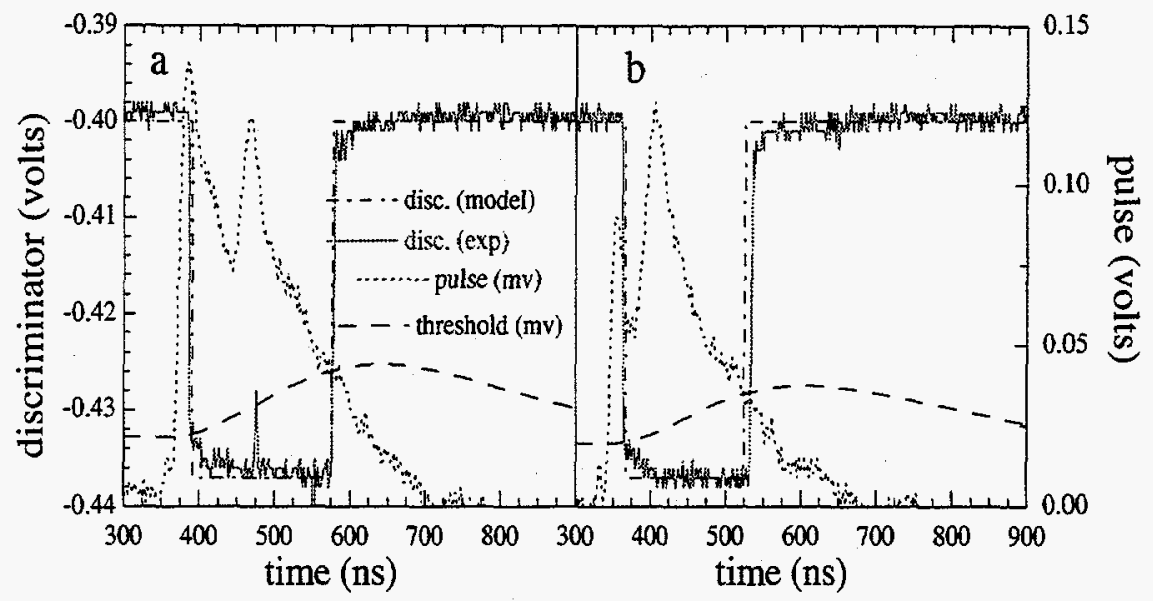

FIG. 9. Response of discriminator model compared with the experimental results. Also shown are the input multiple pulse trains and the movement of the discriminator. Since the TPC drift time was $10000 \mathrm{~ns}$ the threshold was usually on the baseline. a.\& b. correspond to two multiple pulse examples.
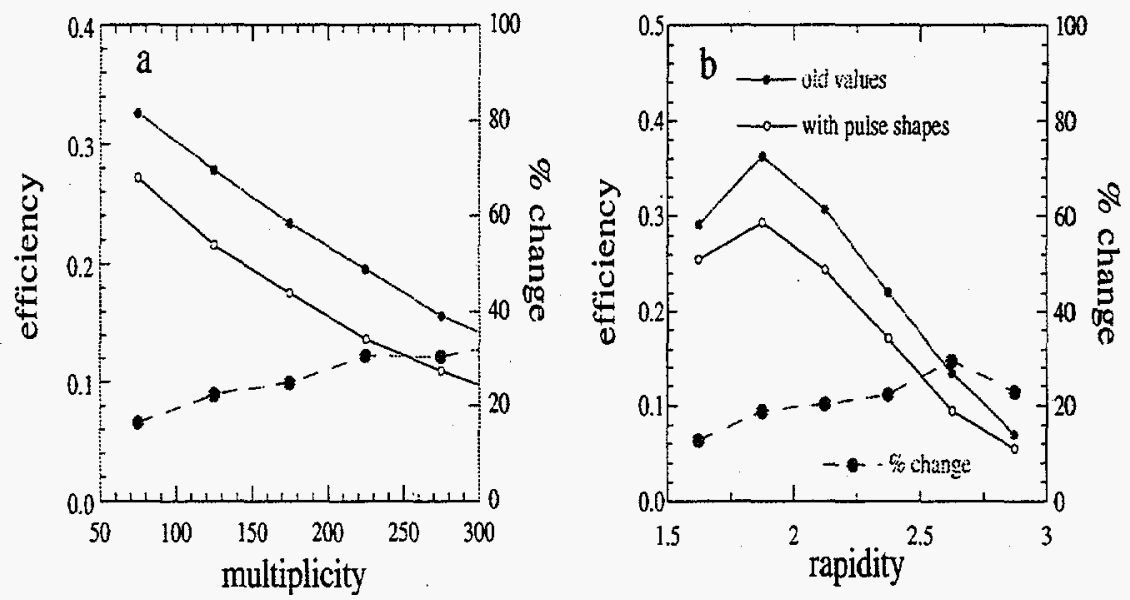

FIG. 10. a. Efficiency to detect $\Lambda$ particles in NA36 S + Pb data as a function of the multiplicity of tracks in the TPC. The old and new values are shown as well as the percent difference. $b$. Same information as a function of the rapidity of the $\Lambda$. 

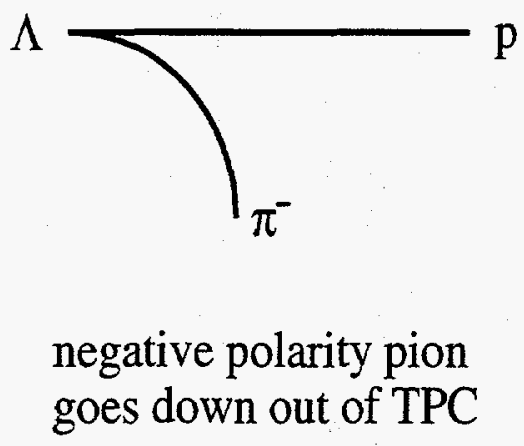

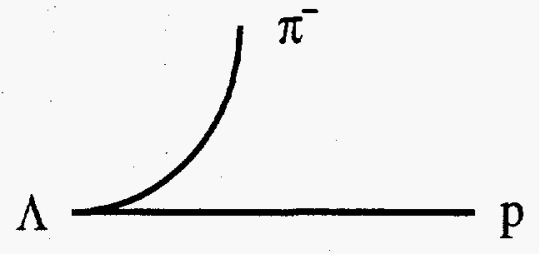

positive polarity pion goes up into TPC

FIG. 11. Topology of $\Lambda$ particles in NA36 TPC for positive and negative polarities of the NA36 magnet. The efficiencies are different because the pion will leave the chamber sooner when it bends down.

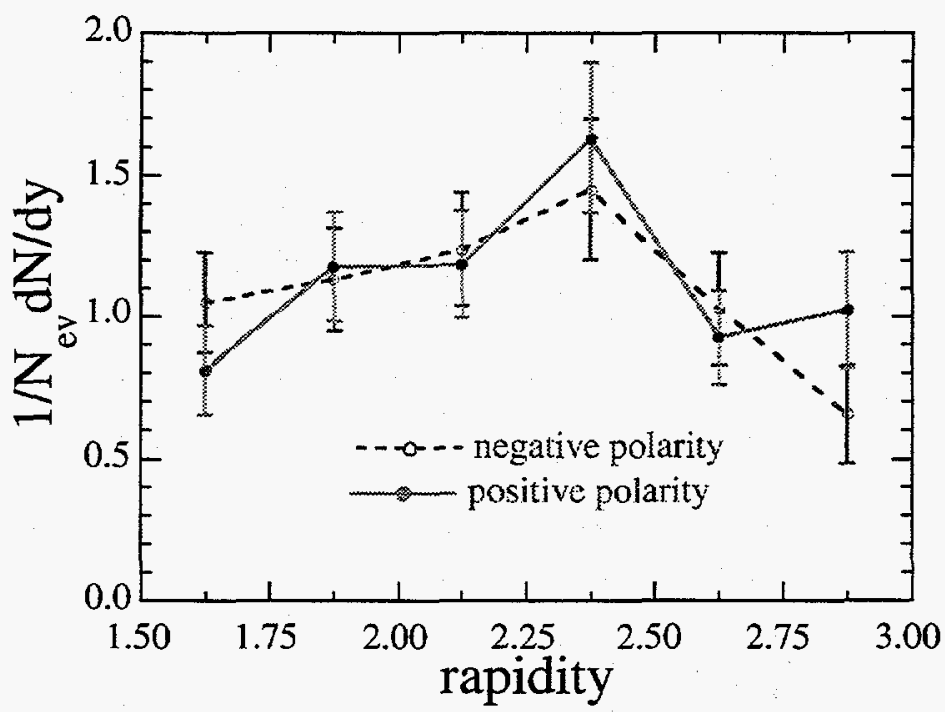

FIG. 12. NA36 $\Lambda$ rapidity spectrum from two independent measurements. One at each polarity of the sweeping magnet. Agreement demonstrates internal consistency of the measurement and analysis. 


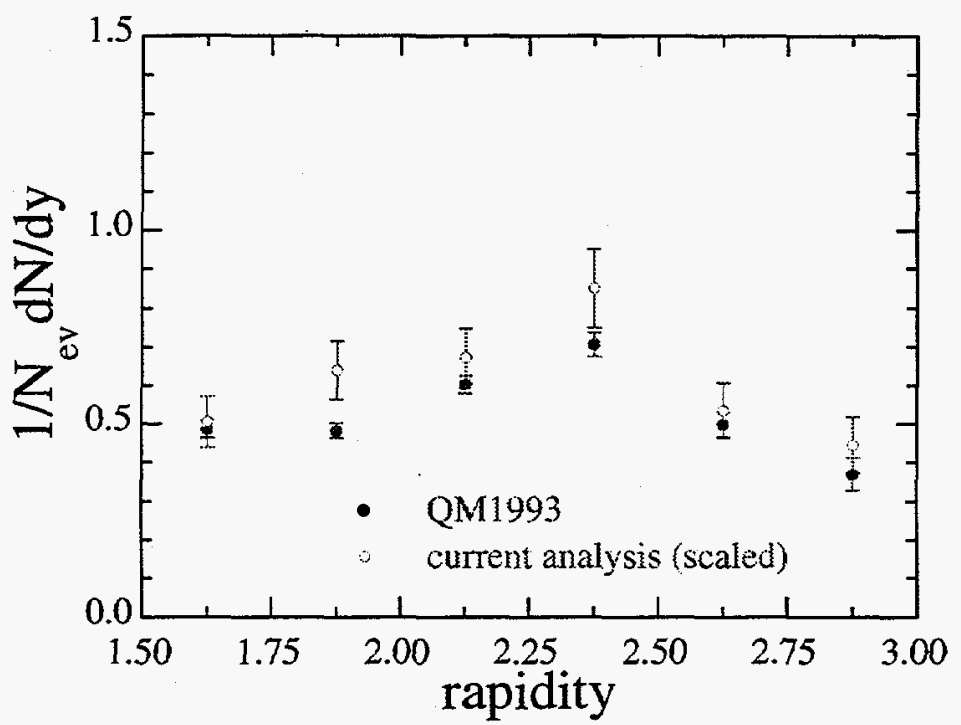

FIG. 13. Comparison of the $\Lambda$ particle rapidity spectrum for data presented at QM93 vs the current analysis of combined positive and negative polarity data. The effect of the revised efficiency calculation based on measured discriminator response can be seen in the average $16 \%$ increased flux. Larger errors result from a re-evaluation of systematic error to be $10 \%$ on individual measurements.

Comparison with models allows us to speculate about the reaction mechanisms. In Fig. 14 we compare with several models. These data are for the $P_{t}$ range $0.4<P_{t}<1.8 \mathrm{GeV} / \mathrm{c}$, trigger is $52 \%$ of the reaction cross section as determined by energy at zero degrees and all model calculations include the contribution from decays of $\Sigma^{0} \Rightarrow \Lambda+\gamma$. FRITIOF 7.0 which has no re-interactions of secondaries is in agreement at the ends of the spectrum and about a factor of two low at the peak near $y=2.4$. This indicates some other mechanisms are needed to explain the data in this region. VENUS 4.02 and RQMD 1.08 have different mechanisms for interaction of secondaries and string-string interactions. We see that VENUS seems to do well for magnitude at the peak and RQMD is about $30 \%$ high there. Both models do not have enough shape change over the range of rapidity measured, though RQMD is at least slightly concave. It appears re-interaction mechanisms may be needed but these present formulations are not yet correct.

If a new mechanism enhancing strangeness becomes active in the more central events one could see it as an increase in the strangeness per negative track as a function of multiplicity. A rise was reported at the low multiplicity region for $\mathrm{S}+\mathrm{S}$ and $\mathrm{S}+\mathrm{Pb}$ at $200 \mathrm{GeV} /$ nucleon $[3,4]$ and is unexplained. These data do not extend to such low multiplicity's. In Fig. 15 we plot the number of $\Lambda$ 's per negative track as a function of the total negative multiplicity in the event. Because of our large trigger range we are able to cover a factor of five in multiplicity. Over this range we see a slight rise but no sharp threshold like increases. Again, seeking insight from the model comparisons the same conclusions may be drawn, FRITIOF is a bit low in general indicating the need for re-interactions while the re-interaction models over predict. The over prediction increases with multiplicity which may be a hint as to the problems with the current mechanisms used.

As one can see from this data NA36 sees about the flux expected from model calculations and thus no great strangeness enhancement. NA35 has reported strangeness enhancement by various measures of a factor of two for reactions of $\mathrm{S}+\mathrm{S}$ and $\mathrm{S}+\mathrm{Ag}$ at $200 \mathrm{GeV} /$ nucleon energies. [5] Such an important result should be corroborated by as many experiments as possible. Different targets, acceptances and triggers make comparisons complicated. Here we will discuss a comparison between the NA36 S + Pb data and results from NA35 for $\mathrm{S}+\mathrm{Ag}$ and $\mathrm{S}+\mathrm{S}$. In Fig. 16 are plotted three points in the $\Lambda$ rapidity spectrum from NA35 for $\mathrm{S}+\mathrm{Ag}$ which are within the NA36 rapidity range. NA35 presents the full phase space values; to compare we need to correct for the range in $P_{t}$, converting to the NA36 region of $0.4<P_{t}<1.8 \mathrm{GeV} / \mathrm{c}$. To do this we use the assumptions that were made by the authors to convert from their acceptance to full phase space, namely that the $P_{t}$ distribution is independent of rapidity and is well expressed by an exponential. Then the conversion factor is:

$$
\frac{\int_{0.4}^{1.8} p_{t} e^{\frac{m_{t}}{T}} d p_{t}}{\int_{0}^{\infty} p_{t} e^{\frac{m_{t}}{T}} d p_{t}=} 0.76 \text { for } \mathrm{T}=234 \mathrm{MeV}
$$



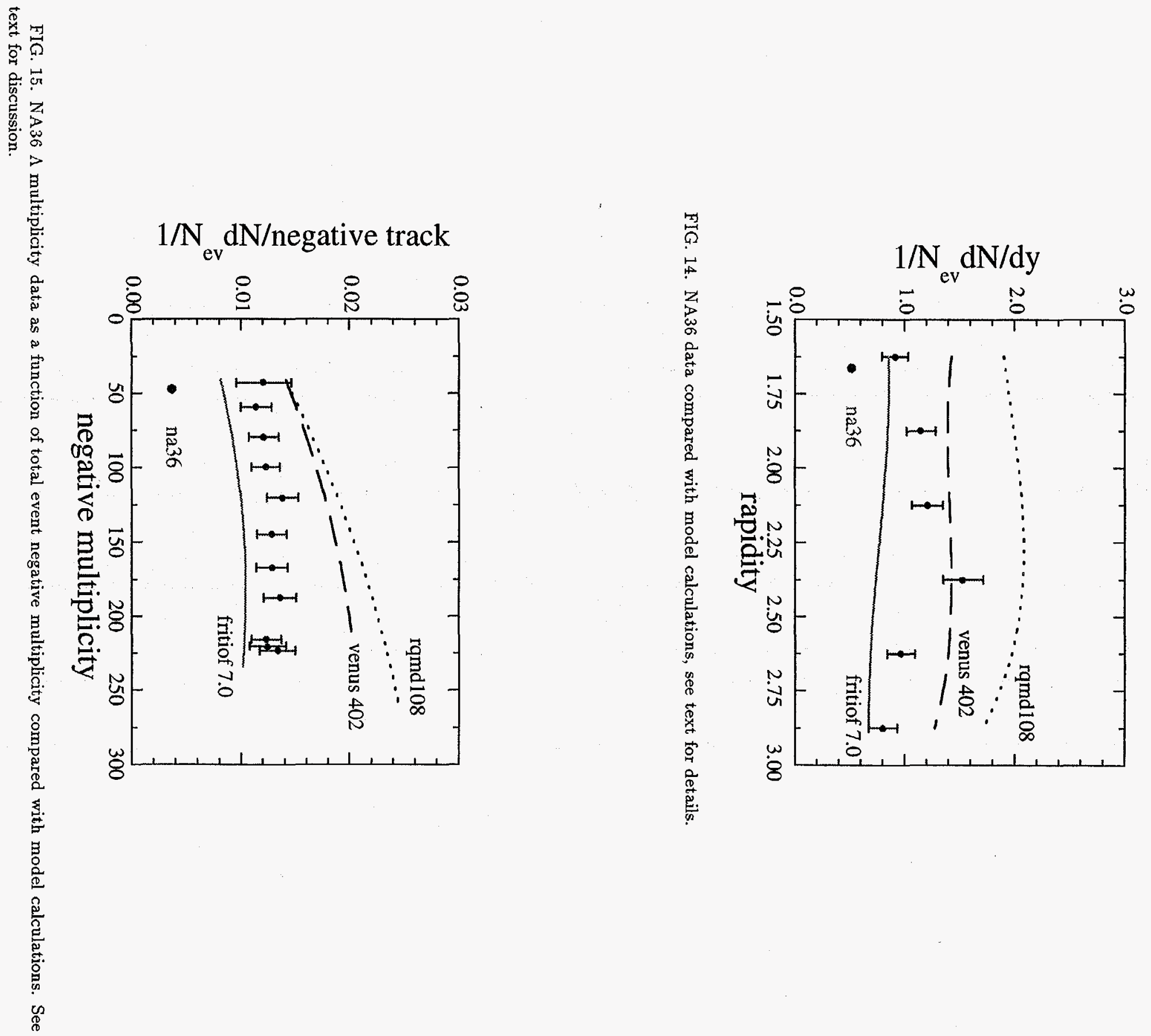


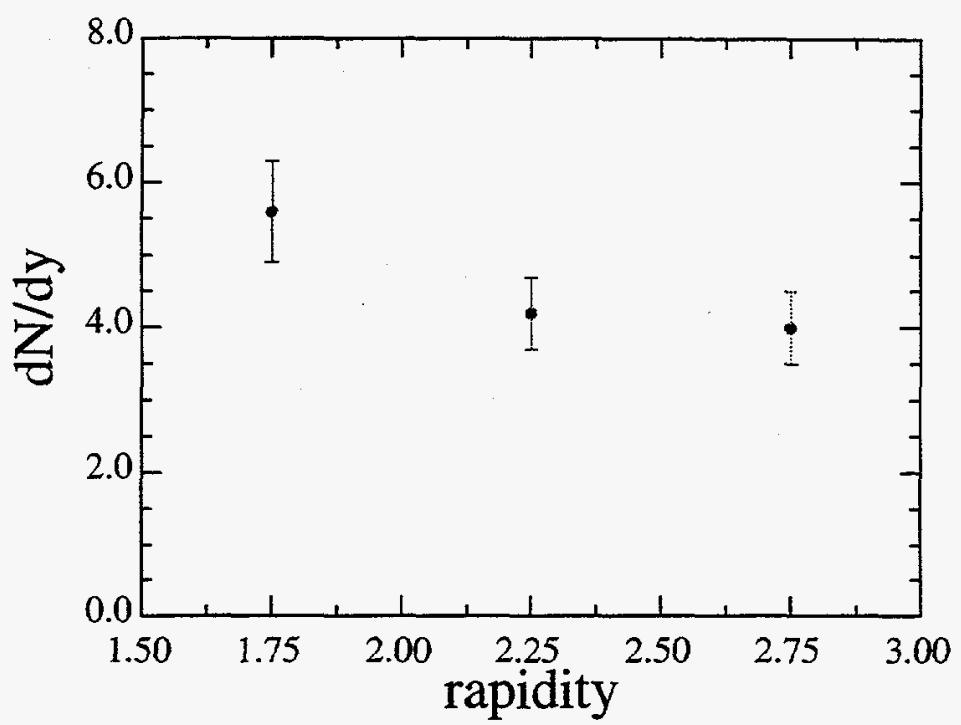

FIG. 16. NA35 $\Lambda$ rapidity distribution in the acceptance of NA36. Data is for $\mathrm{S}+\mathrm{Ag}$ with a trigger corresponding to a negative hadron multiplicity of 170 particles per event. Points are extrapolated to full space using a temperature of $234 \mathrm{MeV}$.

To scale the trigger and target we make the assumption that all reactions are the same if they make the same number of negative hadrons. This ratio is 0.85 for the NA36/NA35 data sets. The comparison in both rapidity and multiplicity is shown in Fig. 17a,b. In general, using the scaling assumptions we have made, we see a disagreement of at least a factor of two at all points. The NA36 experiment has approximately 100 times larger statistics and can thus use more robust methods of signal extraction. NA36 has some $\mathrm{S}+\mathrm{S}$ data that can be directly compared with NA35 results under analysis. [6] The question may need to be resolved by new measurements from different experiments. If NA44 and WA94 could devise triggers that allow a measurement of absolute flux it would provide an independent check of this important question of the existence of strangeness enhancement in nuclear reactions at CERN SPS energies.

While NA36 does not see a large strangeness enhancement there is an unexplained dependence of $\Lambda$ production on multiplicity. This can be seen by looking at the data split into two rapidity ranges. The two ranges are: the region of the peak covering rapidity $2.25<\mathrm{y}<3.25$ and the region before the peak, rapidity $1.25<\mathrm{y}<2.25$. In Fig. 18a we see the multiplicity dependence of the $\Lambda$ /event for these two regions. The effect we wish to point out is that the $\Lambda$ flux for the peak region is always greater and begins to diverge as the multiplicity becomes greater than about 180 negative hadrons per event. To show the unusual nature of this effect we plot in Fig $18 \mathrm{~b}$ the same curves for the VENUS 4.02 model, which exhibits the typical model behavior, here we see that the effect is reversed from what is seen in the data. A possible conclusion is that there is some mechanism that is providing a slight( $30 \%)$ enhancement of $\Lambda$ production near rapidity 2.4 that is not seen in the current forms of re-interaction codes.

\section{CONCLUSIONS}

The NA36 efficiency calculation has been revised to account for new information about the response of the electronics to pulses alone and overlapping in time. This change was an average of $16 \%$ increase of signal for $\Lambda$ production when compared to the values reported at QM93. The spectrum shape is unchanged. The internal consistency of the NA36 data set was demonstrated by agreement of two independent measurements of the $\Lambda$ rapidity distribution. A factor of two disagreement was shown regarding the absolute flux of $\Lambda$ particles between NA35 and NA36 with NA35 seeing the higher value. This brings into question the effect of strangeness enhancement in nuclear collisions at CERN-SPS energies. The NA36 data show an unexplained peak in the $\Lambda$ rapidity distribution at $y=2.4$ which is enhanced at high multiplicity's. 

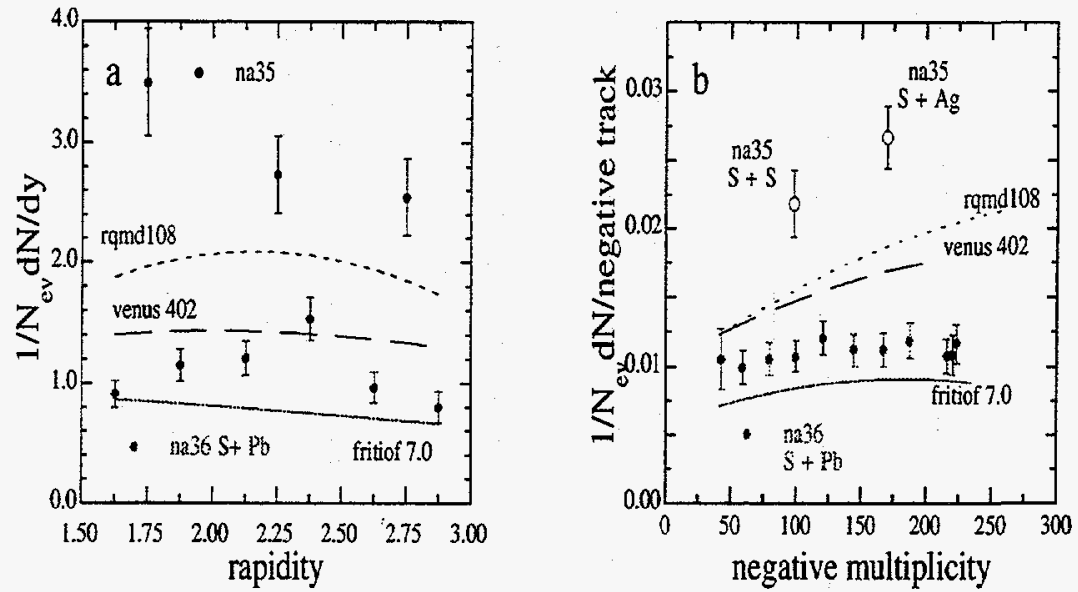

FIG. 17. Comparison of NA36 results for $\mathrm{S}+\mathrm{Pb}$ to NA35 results for $\mathrm{S}+\mathrm{Ag}$ scaled to account for account for trigger and acceptance differences. a. $\Lambda$ rapidity distribution, $b . \Lambda$ /negative track vs event negative multiplicity.
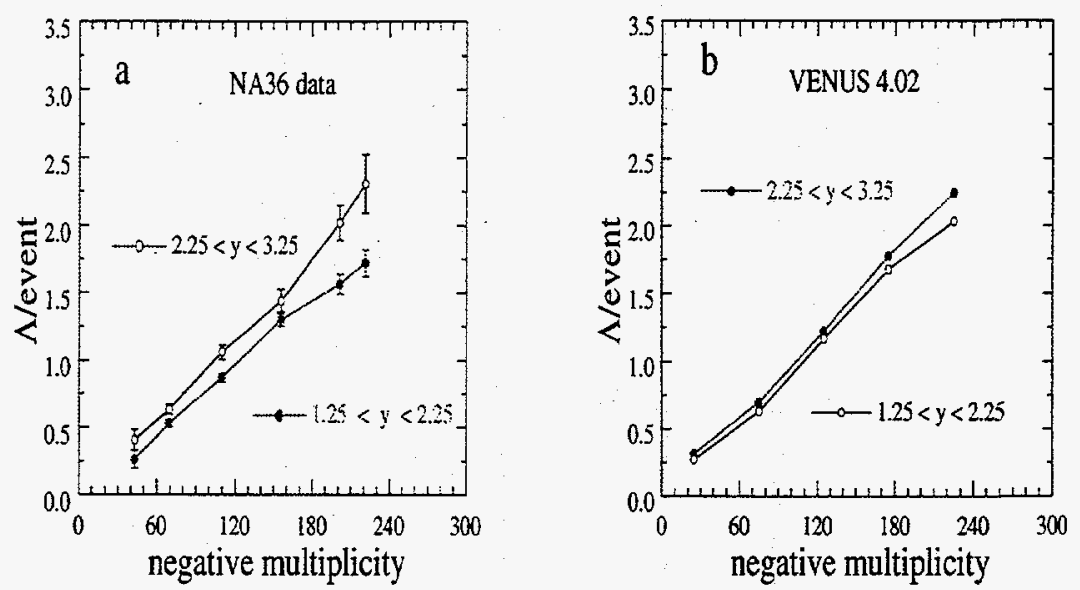

FIG. 18. a. NA36 $\Lambda$ /event as a function of event negative multiplicity for rapidity region $2.25<\mathrm{y}<3.25$ and $1.25<\mathrm{y}<$ 2.25. b. Same plot for the model VENUS 4.02. Note that the NA36 data indicates that the enhancement in flux in the region $2.25<\mathrm{y}<3.25$ for the highest multiplicity's is not predicted by VENUS 4.02 . 


\section{ACKNOWLEDGEMENTS}

Part of this work was supported by EC grant A88000145; Director, Office of Energy Research, Division of Nuclear Physics of the Office of High Energy and Nuclear Physics of the U. S. Department of Energy under contract nos. DE-AC03-76SF00098 and DE-FG02-91ER40652; United Kingdom Science and Engineering Research Council under Grant GR/H 71314; Norway under a grant from Norges Forskningsråd (NAVF) and Spain under CICYT contracts 85-0022, AE86-0031, AE87-0031, AE88-0031, AE89-0589, AE90-0031,AEN91-0739 and XUGA 80409288. Much of the computing work has been done using the IBM PPCS STAGE 2 compute server. The authors are grateful to CERN and IBM for making it available as well as to CERN's PPCS group and especially D.Lord for their help and support in using the PPCS STAGE 2 computer system.

[1] NA36 Collaboration, C. Garabatos et al., Nucl.Instr. and Methods A283 (1989) 553.

[2] Proceedings of the International conference on UltraRelativistic NucleusNucleus collisions (Quark Matter 93), June 2024, 1993, Borlange, Sweden, Nucl. Phys. A211 (1994).

[3] NA35 Collaboration, J. Bartke et al., Z. Phys. C 48 (1990) 191.

[4] E. Andersen, et al., Phys. Lett. 316B (1993) 603.

[5] NA35 Collaboration, T. Alber et al., Z. Phys. C 64 (1994) 195.

[6] E. Judd, the proceedings of the Proceedings of the 12th International conference on Ultra-Relativistic Nucleus-Nucleus collisions (Quark Matter 95), January 9-13,1995, Monterey, California USA, To be published in Nucl.Phys. A 